\title{
Multi-Source Domain Adaptation with Mixture of Experts
}

\author{
Jiang Guo, Darsh J Shah and Regina Barzilay \\ Computer Science and Artificial Intelligence Laboratory \\ Massachusetts Institute of Technology \\ \{jiang_guo, darsh, regina\}ecsail.mit.edu
}

\begin{abstract}
We propose a mixture-of-experts approach for unsupervised domain adaptation from multiple sources. The key idea is to explicitly capture the relationship between a target example and different source domains. This relationship, expressed by a point-to-set metric, determines how to combine predictors trained on various domains. The metric is learned in an unsupervised fashion using metatraining. Experimental results on sentiment analysis and part-of-speech tagging demonstrate that our approach consistently outperforms multiple baselines and can robustly handle negative transfer. ${ }^{1}$
\end{abstract}

\section{Introduction}

Typical domain adaptation methods are designed to transfer supervision from a single source domain. However, in many practical applications, we have access to multiple sources. For instance, in sentiment analysis of product reviews, we can often transfer from a wide range of product domains, rather than one. This can be particularly promising for target domains which do not match any one available source well. For example, the Kitchen product domain may include reviews on pans, cookbooks or electronic devices, which cannot be perfectly aligned to a single source such as Cookware, Books or Electronics. By intelligently aggregating distinct and complementary information from multiple sources, we may be able to better fit the target distribution.

A straightforward approach to utilizing data from multiple sources is to combine them into a single domain. This strategy, however, does not account for distinct relations between individual sources and the target example. Constructing a

\footnotetext{
${ }^{1}$ Our code and data are available at https : / / github . com/jiangfeng1124/transfer.
}

common feature space for this heterogeneous collection may wash out informative characteristics of individual domains and also lead to negative transfer (Rosenstein et al., 2005).

Therefore, we propose to explicitly model the relationship between different source domains and target examples. We hypothesize that different source domains are aligned to different sub-spaces of the target domain. Specifically, in this paper, we model the domain relationship with a mixtureof-experts (MoE) approach (Jacobs et al., 1991b). For each target example, the predicted posterior is a weighted combination of all the experts' predictions. The weights reflect the proximity of the example to each source domain. Our model learns this point-to-set metric automatically, without additional supervision.

We define the point-to-set metric using Mahalanobis distance (Weinberger and Saul, 2009) between individual examples and a set (i.e. domain), which are computed within the hidden representation space of our model. The main challenge is to learn this metric in an unsupervised setting. We address it through a meta-training procedure, in which we create multiple meta-tasks of domain adaptation from the source domains. In each meta-task, we pick one of the source domains as meta-target, and the rest source domains as metasources. By minimizing the loss using the $\mathrm{MoE}$ predictions on meta-target, we are able to learn both the model and the metric simultaneously. To further improve transfer quality, we align the encoding space of our target and source domains via adversarial learning.

We evaluate our approach on sentiment analysis using the benchmark multi-domain Amazon reviews dataset (Chen et al., 2012; Ziser and Reichart, 2017) as well as on part-of-speech (POS) tagging using the SANCL dataset (Petrov and McDonald, 2012). Experiments show that our ap- 
proach consistently improves the adaptation results over the best single-source model and a unified multi-source model. On average, we achieve a $7 \%$ relative error reduction on the Amazon reviews dataset, and a $13 \%$ on the SANCL dataset. Importantly, the POS tagging experiments on the SANCL dataset demonstrate that our method is able to robustly handle negative transfer from unrelated sources (e.g., Twitter) and utilize it effectively to consistently improve performance.

\section{Related Work}

Unsupervised domain adaptation Most existing domain adaptation methods focus on aligning the feature space between source and target domains to reduce the domain shift (Ben-David et al., 2007; Blitzer et al., 2007, 2006; Pan et al., 2010). Our approach is close to the representation learning approaches, such as the denoising autoencoder (Glorot et al., 2011), the marginalized stacked denoising autoencoders (Chen et al., 2012), and domain adversarial networks (Tzeng et al., 2014; Ganin et al., 2016; Zhang et al., 2017; Shen et al., 2018).

In contrast to these previous approaches, however, our approach not only learns a shared representation space that generalizes well to the target domain, but also captures informative characteristics of individual source domains.

Multi-Source domain adaptation The main challenge in using multiple sources for domain adaptation is in learning domain relations. Some approaches assume that all source domains are equally important to the target domain ( $\mathrm{Li}$ and Zong, 2008; Luo et al., 2008; Crammer et al., 2008). Others learn a global domain similarity metric using labeled data in a supervised fashion (Yang et al., 2007; Duan et al., 2009; Yu et al., 2018). Alternatively, Mansour et al. (2009) and Bhatt et al. (2016) utilize unlabeled data of the target domain to find a distribution weighted combination of the source domains or to construct an auxiliary training set of the source domain instances close to the target domain instances. Recent adversarial methods on multi-source domain adaptation (Zhao et al., 2018; Chen and Cardie, 2018) align source domains to the target domains globally, without accounting for the distinct importance of each source with respect to a specific target example.

The work most related to ours is by Kim et al.

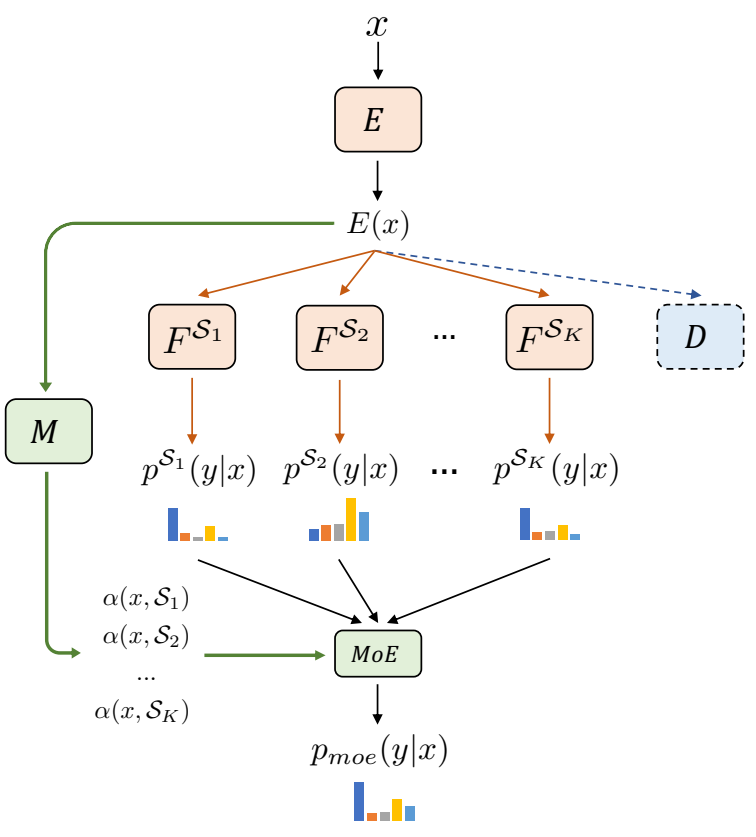

Figure 1: Architecture of the MoE model. $E$ is the encoder which maps an input $x$ to a hidden representation $E(x) ; F^{\mathcal{S}_{i}}$ is the classifier on the $i^{t h}$ source domain; $D$ is the critic that is only used during adversarial training. $M$ is the metric learning component, which takes the encoding of $x$ and source domains $\left(\mathcal{S}_{1: K}\right)$ as input and computes $\alpha$.

(2017). They also model the example-to-domain relations, but use an attention mechanism. The attention module is learned using limited training data from the target domain in a supervised fashion. Our method, however, works in an unsupervised setting without utilizing any labeled data from the target domain.

\section{Methodology}

Problem definition We follow the unsupervised multi-source domain adaptation setup, assuming access to labeled training data from $K$ source domains: $\left\{\mathcal{S}_{i}\right\}_{i=1}^{K}$ where $\mathcal{S}_{i} \triangleq\left\{\left(x_{t}^{\mathcal{S}_{i}}, y_{t}^{\mathcal{S}_{i}}\right)\right\}_{t=1}^{\left|\mathcal{S}_{i}\right|}$, and (optionally) unlabeled data from a target domain: $\mathcal{T} \triangleq\left\{x_{t}^{\mathcal{T}}\right\}_{t=1}^{|\mathcal{T}|}$. The goal is to learn a model using the source domain data, that generalizes well to the target domain.

Notations For the rest of the paper, we denote an individual example as $x$, and a batch of examples as $\mathbf{x}$. We use superscript to denote the domain from which an example is sampled, and use subscript to denote the index of an example. 


\subsection{Overview of Our Approach}

We model the multiple source domains as a mixture of experts, and learn a point-to-set metric $\alpha$ to weight the experts for different target examples. The metric is learned in an unsupervised manner.

Our model consists of four key components as shown in Figure 1, namely the encoder $(E)$, classifier $(F)$, metric $(M)$ and adversary $(D)$. We use a typical neural multi-task learning architecture (Caruana, 1997), with a shared encoder across all sources, and domain-specific classifiers $\left(\left\{F^{\mathcal{S}_{i}}\right\}_{i=1}^{K}\right)$. Each input is first encoded with $E$, and then fed to each classifier to obtain the domain-specific predictions (i.e. posteriors). The final predictions are then weighted based on the metric (see Equation 1).

We start by describing the representation learning component.

\subsection{Representation}

Our goal is to design an encoder that supports transfer, while maintaining source domainspecific information. Depending on different tasks and datasets, we select appropriate encoders MLP, CNN or LSTM (see Section 4.3 for details).

We further add an adversarial module $(D)$ on top of the encoder, in order to align the target domain with the sources. $D$ is typically designed as a parameterized classifier in domain adversarial networks (Ganin et al., 2016; Zhang et al., 2017), which is trained jointly with the encoder and the classifiers through a minimax game. Here, we instead use Maximum Mean Discrepancy (MMD) (Gretton et al., 2012) as our adversary. This distance metric measures the discrepancy between two distributions explicitly in a non-parametric manner, greatly simplifying the training procedure compared to domain adversarial networks which use an additional domain classifier module.

\subsection{Mixture of Experts}

Given an example $x$ from the target domain, we model its posterior distribution as a mixture of posteriors produced by models trained on different source domain data:

$$
\begin{aligned}
p_{\text {moe }}(y \mid x) & =\sum_{i=1}^{K} \alpha\left(x, \mathcal{S}_{i}\right) \cdot p^{\mathcal{S}_{i}}(y \mid x) \\
& =\sum_{i=1}^{K} \alpha\left(x, \mathcal{S}_{i}\right) \cdot \operatorname{sof} \operatorname{tmax}\left(\mathbf{W}^{\mathcal{S}_{i}} E(x)\right)
\end{aligned}
$$

$p^{\mathcal{S}_{i}}$ is the posterior distribution produced by the $i^{\text {th }}$ source classifier $F^{\mathcal{S}_{i}}$ (the $i^{\text {th }}$ expert). $\mathbf{W}^{\mathcal{S}_{i}}$ is the output layer weights of $F^{\mathcal{S}_{i}}, \alpha$ is a parameterized metric function that measures how much confidence we put in the specific source expert for a given example $x .^{2}$ To derive $\alpha$, we first define a point-to-set Mahalanobis distance metric between an example $x$ and a set $\mathcal{S}$ :

$$
d(x, \mathcal{S})=\left(\left(E(x)-\mu^{\mathcal{S}}\right)^{\top} \mathbf{M}^{\mathcal{S}}\left(E(x)-\mu^{\mathcal{S}}\right)\right)^{\frac{1}{2}}
$$

where $\mu^{\mathcal{S}}$ is the mean encoding of $\mathcal{S}$. In its original form, the matrix $\mathbf{M}^{\mathcal{S}}$ played the role of the inverse covariance matrix. However, computing the inverse of the covariance matrix is both time consuming and numerically unstable in practice. Here we allow $\mathbf{M}$ to denote any positive semi-definite matrix which is to be estimated during training (Weinberger and Saul, 2009). To guarantee the positive semi-definiteness of $\mathbf{M}$, we approximate $\mathbf{M}$ with $\mathbf{M}=\mathbf{U U}^{\top}$, where $\mathbf{U} \in \mathbb{R}^{h \times r}, h$ is the dimension of hidden representations and $r$ is a hyper-parameter controlling the rank of $\mathbf{M}$.

Based on the distance metric, we further derive a confidence score $e\left(x, \mathcal{S}_{i}\right)=f\left(d\left(x, \mathcal{S}_{i}\right)\right)$ for each specific expert. The final metric values $\alpha\left(x, \mathcal{S}_{i}\right)$ are then obtained by normalizing these confidence scores:

$$
\alpha\left(x, \mathcal{S}_{i}\right)=\frac{\exp \left(e\left(x, \mathcal{S}_{i}\right)\right)}{\sum_{j=1}^{K} \exp \left(e\left(x, \mathcal{S}_{j}\right)\right)}
$$

Here, we explain our design of $e(x, \mathcal{S})$ on two tasks, respectively binary classification and sequence tagging, which are also used for evaluation in this paper (Section 4).

Binary classification The point-to-set Mahalanobis distance metric measures the distance between an example $x$ and the mean encoding of $\mathcal{S}$, i.e. $\mu^{\mathcal{S}}$, while taking into account the (pseudo) covariance of $\mathcal{S}$. In binary classification, however, the mean vector $\mu^{\mathcal{S}}$ is likely to be located near the decision boundary, particularly under a balanced

\footnotetext{
${ }^{2}$ In traditional MoE frameworks (Jacobs et al., 1991a,b; Shazeer et al., 2017), $\alpha$ is commonly realized as a "gating network", which produces a normalized weight vector that determines the combination of experts depending solely on the input example $x$. However, simple gating networks do not yield promising results in our scenario. We hypothesize that both the input instance and the underlying domain distribution should be captured for determining the credit assignment.
} 
setting. Therefore, a small $d(x, \mathcal{S})$ actually implies lower confidence of the corresponding classifier, which is counter-intuitive. To this end, we instead define the confidence $e(x, \mathcal{S})$ as the difference between the distances from $x$ to each category of $\mathcal{S}$ :

$$
e(x, \mathcal{S})=\left|d\left(x, \mathcal{S}^{+}\right)-d\left(x, \mathcal{S}^{-}\right)\right|
$$

Here $\mathcal{S}^{+}$and $\mathcal{S}^{-}$stand for the positive space and negative space of $\mathcal{S}$ respectively. Consequently, if $x$ is either far away from $\mathcal{S}$ (i.e., $x$ is not in the manifold of $\mathcal{S}$ ) or near the classification boundary, we will get a small $e(x, \mathcal{S})$ indicating a low confidence to the corresponding prediction. On the contrary, if $x$ is much closer to a specific category of $\mathcal{S}$ than other categories, the classifier will get a higher confidence.

Sequence tagging For sequence tagging tasks (e.g., POS tagging), we compute the distance metric at the token level. ${ }^{3}$ Unlike in binary classification, the decision boundary here is more complicated, and the label distribution is typically imbalanced. The mean vector $\mu^{\mathcal{S}}$ is unlikely to be located at the decision boundary. So we directly use the (reverse) distance as the confidence value for each token $x$ :

$$
e(x, \mathcal{S})=-d(x, \mathcal{S})
$$

\subsection{Training}

Since we do not have annotated data in the target domain, we have to learn our model in an unsupervised fashion. Inspired by the recent progress on few-shot learning with metric-based models such as matching network (Vinyals et al., 2016; Yu et al., 2018) and prototypical network (Snell et al., 2017), we propose the following meta-training approach. Given $K$ source domains, each source domain will be considered as a target, referred to as meta-target, with the rest of the source domains as meta-sources. This way, we obtain $K$ (meta-sources, meta-target) training pairs for domain adaptation. Then, we apply our MoE formulation over these meta-training pairs to learn the metric. At testing time, the metric will be applied to all the $K$ source domains for each example in the target domain.

We optimize two main objectives: the MoE objective and the multi-task learning (MTL) objective.

\footnotetext{
${ }^{3}$ This actually makes it a multi-class classification problem with respect to every token of a sequence.
}

MoE objective For each example in each metatarget domain, we compute its MoE posterior using the corresponding meta-sources. Therefore, we get the following MoE loss over the entire multi-source training data:

$$
\begin{aligned}
\mathcal{L}_{\text {moe }} & =-\sum_{i=1}^{K} \sum_{j=1}^{\left|\mathcal{S}_{i}\right|} \log p_{\text {moe }}\left(y_{j}^{\mathcal{S}_{i}} \mid x_{j}^{\mathcal{S}_{i}}\right) \\
& =-\sum_{i=1}^{K} \sum_{j=1}^{\left|\mathcal{S}_{i}\right|} \log \sum_{l=1, l \neq i}^{K} \alpha\left(x, \mathcal{S}_{l}\right) \cdot p^{\mathcal{S}_{l}}\left(y_{j}^{\mathcal{S}_{i}} \mid x_{j}^{\mathcal{S}_{i}}\right)
\end{aligned}
$$

Note that $\alpha$ is normalized over the meta-sources for each meta-target, rather than over all the $K$ sources.

MTL objective For each meta-target, we further optimize a supervised cross-entropy loss using the corresponding labels. All supervised objectives are optimized jointly with the encoder being shared, resulting in the following multi-task learning objective:

$$
\mathcal{L}_{m t l}=-\sum_{i=1}^{K} \sum_{j=1}^{\left|\mathcal{S}_{i}\right|} \log p^{\mathcal{S}_{i}}\left(y_{j}^{\mathcal{S}_{i}} \mid x_{j}^{\mathcal{S}_{i}}\right)
$$

Adversary-augmented MoE We use MMD (Gretton et al., 2012) as the adversary to minimize the divergence between the marginal distribution of target domain and source domains. Specifically, at each training epoch, given the $K$ batches $\left\{\mathbf{x}^{\mathcal{S}_{1}}, \mathbf{x}^{\mathcal{S}_{2}}, \ldots, \mathbf{x}^{\mathcal{S}_{K}}\right\}$ from all the source domains, we sample a batch (unlabeled) $\mathrm{x}^{\mathcal{T}}$ from our target domain, and minimize the MMD:

$$
\mathcal{L}_{a d v}=\operatorname{MMD}^{2}\left(\mathbf{x}^{\mathcal{S}_{1}} \cup \ldots \cup \mathbf{x}^{\mathcal{S}_{K}}, \mathbf{x}^{\mathcal{T}}\right)
$$

where

$$
\begin{aligned}
& \operatorname{MMD}\left(\mathcal{D}^{\mathcal{S}}, \mathcal{D}^{\mathcal{T}}\right)= \\
& \left\|\frac{1}{\left|\mathcal{D}^{\mathcal{S}}\right|} \sum_{x_{s} \in \mathcal{D}^{\mathcal{S}}} \phi\left(E\left(x_{s}\right)\right)-\frac{1}{\left|\mathcal{D}^{\mathcal{T}}\right|} \sum_{x_{t} \in \mathcal{D}^{\mathcal{T}}} \phi\left(E\left(x_{t}\right)\right)\right\|_{\mathcal{H}}
\end{aligned}
$$

measures the discrepancy between $\mathcal{D}^{\mathcal{S}}$ and $\mathcal{D}^{\mathcal{T}}$ based on Reproducing Kernel Hilbert Space (RKHS). $\phi(\cdot)$ is the feature map induced by a universal kernel. We follow Bousmalis et al. (2016) and use a linear combination of multiple RBF kernels: $\kappa\left(\boldsymbol{h}_{i}, \boldsymbol{h}_{j}\right)=\Sigma_{n} \exp \left(-\frac{1}{2 \sigma_{n}}\left\|\boldsymbol{h}_{i}-\boldsymbol{h}_{j}\right\|^{2}\right)$. 


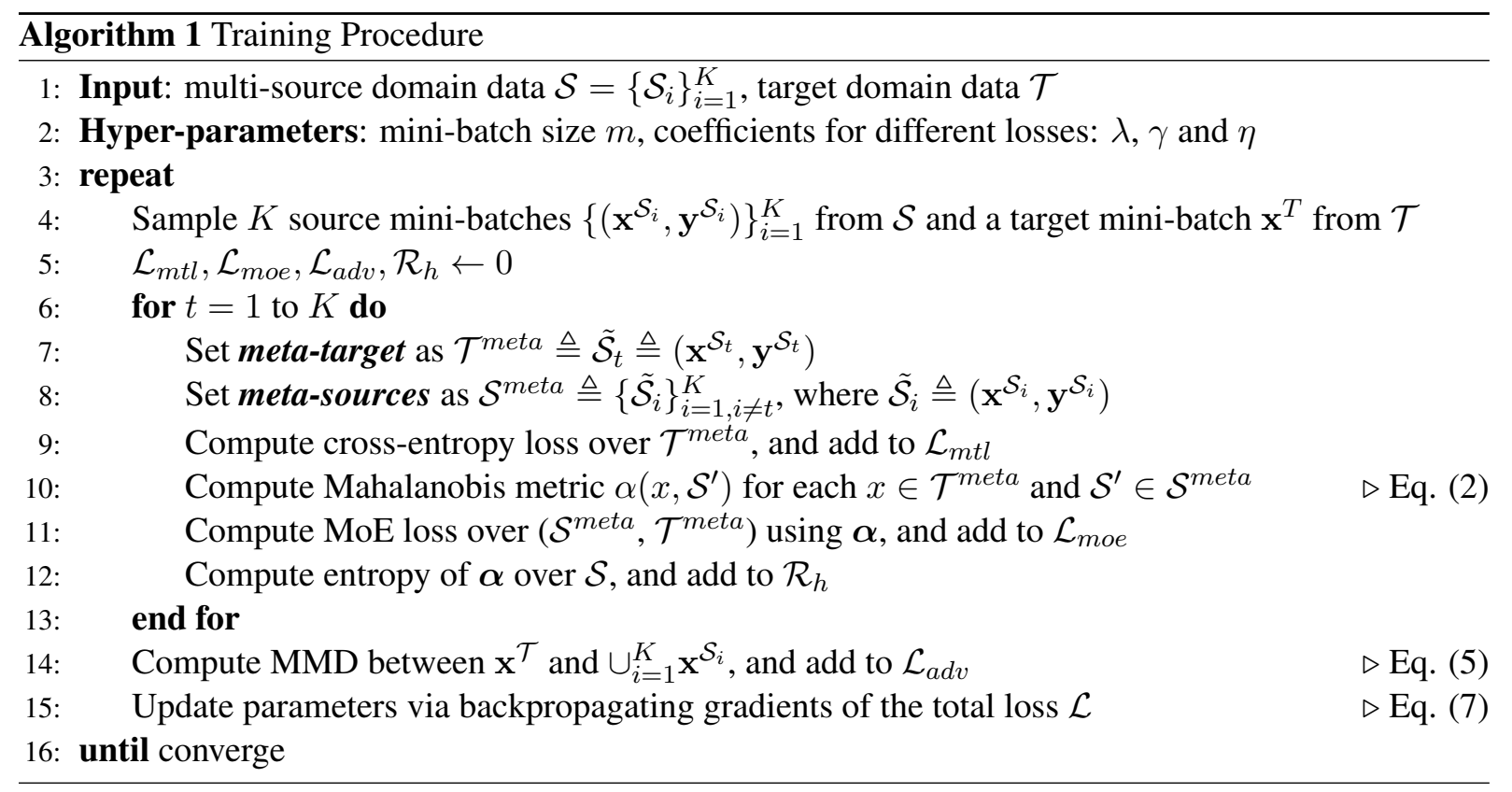

Entropy regularization In the meta-training process, for each example $x$ in meta-target, we know exactly from which source $x$ is sampled. This provides additional insight that the $\alpha$ distribution is skewed, which can be utilized as a soft constraint. Therefore, we propose to regularize the entropy of the $\alpha$ distribution over all the sources, rather than meta-sources: ${ }^{4}$

$$
\begin{gathered}
H(\boldsymbol{\alpha}(x, \cdot))=-\sum_{l=1}^{K} \alpha\left(x, \mathcal{S}_{l}\right) \cdot \log \alpha\left(x, \mathcal{S}_{l}\right) \\
\mathcal{R}_{h}=\sum_{i=1}^{K} \sum_{j=1}^{\left|\mathcal{S}_{i}\right|} H\left(\boldsymbol{\alpha}\left(x_{j}^{\mathcal{S}_{i}}, \cdot\right)\right)
\end{gathered}
$$

Joint learning Our final objective is the weighted combination of each individual component loss:

$$
\begin{aligned}
\mathcal{L}= & \lambda \cdot \mathcal{L}_{m o e}+(1-\lambda) \cdot \mathcal{L}_{m t l} \\
& +\gamma \cdot \mathcal{L}_{a d v} \\
& +\eta \cdot \mathcal{R}_{h}
\end{aligned}
$$

where $\lambda$ controls the balance of the MoE loss and MTL loss. $\gamma$ is set to 0 in non-adversarial setting when unlabeled data from the target domain

\footnotetext{
${ }^{4}$ Alternatively, we can directly exploit this supervision and minimize the KL divergence of the $\alpha$ distribution and its ground truth one-hot distribution. In practice, however, we found it beneficial to allow examples from one domain to be attended to different sources. This observation may be attributed to the fact that each domain indeed consists of multiple latent sub-domains.
}

is not provided. Additionally, it would be straightforward to add an MoE loss for labeled data in the target domain if they are available, thus extending our framework to a setting where we have fewshot target annotations. The training process is shown in Algorithm 1.

\section{Experimental Setup}

\subsection{Task and Dataset}

Sentiment classification We use the multidomain Amazon reviews dataset (Blitzer et al., 2007), one of the standard benchmark datasets for domain adaptation. It contains reviews on four domains: Books (B), DVDs (D), Electronics (E), and Kitchen appliances $(\mathrm{K})$.

We follow the specific experiment settings proposed by Chen et al. (2012) (CHEN12) and Ziser and Reichart (2017) (ZISER17).

1. In CHEN12, each domain has 2,000 labeled examples for training (1,000 positive and 1,000 negative), and the target test set has 3,000 to 6,000 examples. $^{5}$

2. In ZISER 17, each domain also has 2,000 labeled examples $(1,000$ positive and 1,000 negative), sampled differently from CHEN12.

For each dataset, we conduct experiments by selecting the target domain in a round-robin fash-

\footnotetext{
${ }^{5}$ This dataset has been processed by the author to TF-IDF representations, using the 5,000 most frequent unigram and bigram features, thus word order information is not available.
} 
ion. Following the protocol in previous work, we use cross-validation over source domains for hyper-parameters selection for each adaptation task (Zhao et al., 2018). When training with an adversary, we use the 2,000 examples training set of the target domain as the unlabeled data in both the settings. In ZISER 17, the same data is also used for test, resulting in a transductive setting.

Part-of-Speech tagging We further consider a sequence tagging task, where the metric is computed over the token-level encodings and multiclass predictions are made at the token (word) level. We use the SANCL dataset (Petrov and McDonald, 2012) which contains part-of-speech (POS) tagging annotations in 5 web domains: Emails, Weblogs, Answers, Newsgroups, and Reviews. Among these, Newsgroups, Reviews, and Answers have both a validation and a test set, and are used as target domains. The test set from $\mathrm{We}$ blogs and Emails are used as individual source domains. The tagging is performed using the Universal POS tagset (Petrov et al., 2012). We also use Twitter (Liu et al., 2018) as an additional training source. Since it differs substantially from other sources and the target domain, we can assess our model's ability to handle negative transfer. We consider 750 sentences from each SANCL source domain for training, and up to 2,250 sentences from the Twitter dataset to magnify the negative transfer. The validation set in the standard split of each target domain is used for hyper-parameters selection and early-stopping in our experiments.

\subsection{Baselines}

We verify the efficacy of our approach (MoE) in non-adversarial and adversarial settings respectively. In both settings, we compare our approach against the following two baselines:

- best-SS: the best single-source adaptation model among all the sources.

- uni-MS: the unified multi-source adaptation model, which is trained using the combination of all the source domain data with singlesource transfer methods. uni-MS is a common and strong baseline for multi-source domain adaptation (Zhao et al., 2018).

For the rest of the paper, we name the adversarial counterpart of the models as $*-\mathbf{A}$.

In the adversarial setting on CHEN12, in addition to best-SS and uni-MS with adversarial loss, we further compare with the following two systems that also utilize unlabeled data from target domain.

- mSDA: the marginalized stacked denoising autoencoder (Chen et al., 2012). mSDA outperforms prior deep learning and shallow learning approaches such as structural correspondence learning (Blitzer et al., 2007) and denoising autoencoder (Glorot et al., 2011).

- MDAN: the multi-source domain adversarial network (Zhao et al., 2018). MDAN gives the state-of-the-art performance for multi-source domain adaptation on CHEN12. It generalizes the domain adversarial network to multiple source domain adaptation by selectively backpropagating the domain discrimination loss according to domain classification error.

\subsection{Implementation Details}

For CHEN12, since the dataset is in TF-IDF format and the word ordering information is not available, we use a multilayer perceptron (MLP) with an input layer of 5,000 dimensions and one hidden layer of 500 dimensions as our encoder. For ZISER 17, we instead use a convolutional neural network $(\mathrm{CNN})$ encoder with a combination of kernel widths 3 and 5 (Kim, 2014), each with one hidden layer of size 150, which are then concatenated to a 300 dimension representation. ${ }^{6}$

For the POS tagging encoder, we use a hierarchical bidirectional LSTM (BiLSTM) network, which contains a character-level BiLSTM for generating individual word representations, followed by a word-level BiLSTM that generates contextualized word representations.

For MMD, we follow Bousmalis et al. (2016) and use 19 RBF kernels with the standard deviation parameters ranging from $10^{-6}$ to $10^{6}$.

All the models were trained using Adam with weight decay. Learning rate is set to $10^{-4}$ for CHEN 12 and $10^{-3}$ for ZISER 17 and POS tagging. We use mini-batches of 32 samples from each domain. We tune the coefficients $\lambda, \eta$ for each adaptation task. $\gamma$ is set to 1 for all experiments.

\footnotetext{
${ }^{6}$ Note that with a more extensive architecture search, we are likely to achieve better results. This, however, is not the main focus of this work.

${ }^{7}$ Detailed values are presented in the supplementary material in Bousmalis et al. (2016).
} 


\begin{tabular}{|c|c|c|c|c|c|c|c|c|}
\hline \multirow{2}{*}{ SETTING } & \multicolumn{3}{|c|}{ NON-ADVERSARIAL } & \multicolumn{5}{|c|}{ ADVERSARIAL } \\
\hline & best-SS & uni-MS & $\mathrm{MoE}$ & $\mathrm{mSDA}^{\dagger}$ & MDAN & best-SS-A & uni-MS-A & MoE-A \\
\hline $\mathrm{D}, \mathrm{E}, \mathrm{K}-\mathrm{B}$ & 75.43 & 78.43 & 79.42 & 76.98 & 78.63 & 80.07 & 80.25 & 80.87 \\
\hline $\mathrm{B}, \mathrm{E}, \mathrm{K}-\mathrm{D}$ & 81.23 & 82.49 & 83.35 & 78.61 & 80.65 & 82.68 & 83.30 & 83.99 \\
\hline $\mathrm{B}, \mathrm{D}, \mathrm{K}-\mathrm{E}$ & 85.51 & $84.79^{*}$ & 86.62 & 81.98 & 85.34 & 86.32 & $85.96^{*}$ & 86.38 \\
\hline $\mathrm{B}, \mathrm{D}, \mathrm{E}-\mathrm{K}$ & 86.83 & 87.00 & 87.96 & 84.33 & 86.26 & 87.05 & 87.55 & 88.06 \\
\hline Average & 82.25 & 83.18 & 84.34 & 80.48 & 82.72 & 84.03 & 84.27 & 84.83 \\
\hline
\end{tabular}

Table 1: Multi-Source domain adaptation accuracy on Amazon dataset of CHEN12. * indicates negative transfer, i.e., the unified multi-source model underperforms the best single-source model. $\mathrm{mSDA}^{\dagger}$ is not an adversarial approach, but utilizes unlabeled data from target domain.

\begin{tabular}{lccccccc}
\hline \multirow{2}{*}{ SETTING } & \multicolumn{3}{c}{ NON-ADVERSARIAL } & & \multicolumn{3}{c}{ ADVERSARIAL } \\
\cline { 2 - 3 } \cline { 7 - 8 } & best-SS & uni-MS & MoE & & best-SS-A & uni-MS-A & MoE-A \\
\hline D,E,K-B & 85.35 & 87.00 & $\mathbf{8 7 . 5 5}$ & & 86.85 & 87.55 & $\mathbf{8 7 . 8 5}$ \\
B,E,K-D & 85.25 & 86.80 & $\mathbf{8 7 . 8 5}$ & & 86.00 & 87.40 & $\mathbf{8 7 . 6 5}$ \\
B,D,K-E & 86.80 & 88.30 & $\mathbf{8 9 . 2 0}$ & & 88.90 & 89.35 & $\mathbf{8 9 . 5 0}$ \\
B,D,E-K & 88.90 & 89.65 & $\mathbf{9 0 . 4 5}$ & & 89.95 & 90.35 & $\mathbf{9 0 . 4 5}$ \\
\hline Average & 86.58 & 87.94 & $\mathbf{8 8 . 7 6}$ & & 87.93 & 88.66 & $\mathbf{8 8 . 8 6}$ \\
\hline
\end{tabular}

Table 2: Multi-Source domain adaptation accuracy on Amazon dataset of ZISER17.

\section{Results}

\subsection{Sentiment Analysis on Amazon Reviews}

We report our results on the Amazon reviews datasets in Table 1 (CHEN12) and Table 2 (ZISER 17). Our approach (MoE) consistently achieves the best performance across different settings and tasks.

The results clearly demonstrate the value of using multiple sources. In most cases, even a unified model performs better than the oracle best single source. By smartly combining all the sources, our model outperforms the unified model significantly. One exception is the task of "B,D,KE" in Chen12, where the unified multi-source model doesn't improve over the best single source model, constituting a negative transfer scenario. However, even in this scenario, our approach still performs significantly better, demonstrating its robustness in handling negative transfer.

Impact of adversarial adaptation We achieve consistent improvements over the baseline systems with the addition of the adversarial loss. In most cases, MoE also achieves additional improvement (e.g., $79.42 \%$ vs. $80.87 \%$ in "D,E,KB"). We notice that in some cases, e.g., "B,D,KE" in CHEN12 and "B,E,K-D" in ZISER 17, the adversarial loss doesn't help MoE. This might be attributed to the fact that by aligning the target distribution with the source domains, the representation space becomes more compact, thus making it more difficult to capture source domain-specific characteristics and increasing the difficulty of metric learning in MoE.

Analysis on the metric $(\alpha)$ Figure 2 visualizes the distribution of $\alpha$ values, learned by our model in different tasks, across the source domains. The visualization is based on 200 examples for each domain randomly sampled from the corresponding test set. From the heatmap we can see that for a specific target domain, different examples may have different $\alpha$ distributions. Moreover, for most examples, the $\alpha$ distribution is skewed, indicating that our model draws on a few most informative source domains.

Figure 3 exemplifies the above point. For instance, the first review about "charger" and "battery" is closer to the Electronics source domain. This relation is successfully captured by the $\alpha$ distribution produced by our model.

We further investigate the impact of entropy regularization over $\alpha$. Table 3 summarizes the ablation test results of entropy regularization $(\eta=0)$ 

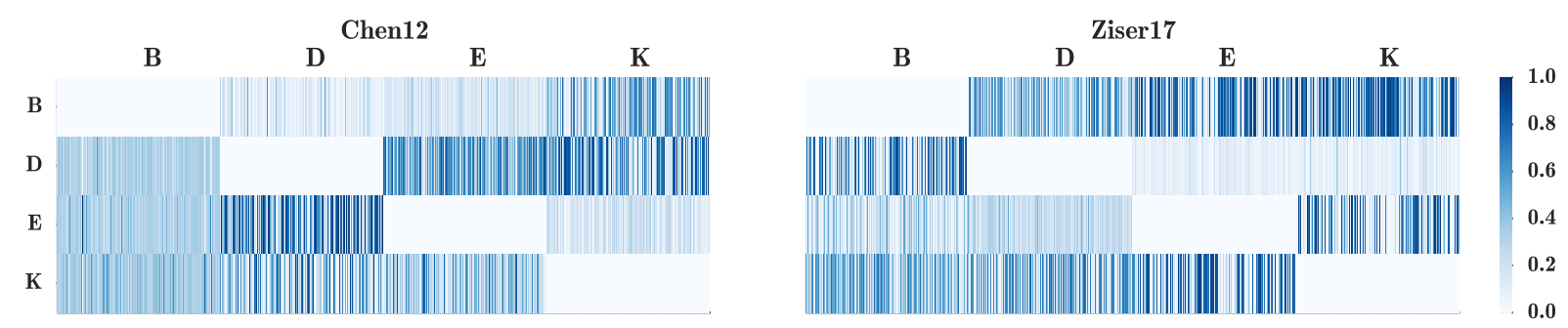

Figure 2: $\alpha$ distributions across source domains for randomly selected 200 examples in each target domain of CHEN12 (left) and ZISER 17 (right). Columns represent target domains and rows represent sources.

- ... i did n't keep it on the charger for 24 hours ( not realizing that this is a problem ) and now the battery only works for 10 minutes at a time ...

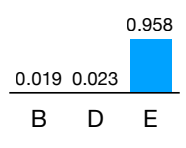

- best 2 quart pot in the world .... with the glorious one pot meals cookbook . it has wonderful recipies, and the pot works wonderful .

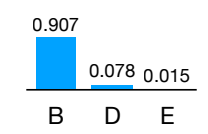

- great kit however the book that comes with the kit needs some work. the photos in the book are not accurate with the descriptions....

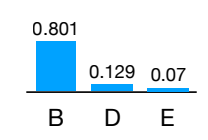

Figure 3: Examples of Kitchen (K) reviews in ZISER 17 and their $\alpha$ distribution over Books (B), DVDs (D) and Electronics (E). The manually highlighted words indicate the specific Kitchen products described in the reviews.

\begin{tabular}{cccccc}
\hline \multirow{2}{*}{ SetTing } & \multicolumn{2}{l}{ Chen12 $\left(\right.$ w/o $\left.\mathcal{R}_{h}\right)$} & & \multicolumn{2}{l}{ Ziser 17 $\left(\right.$ w/o $\left.\mathcal{R}_{h}\right)$} \\
\cline { 2 - 3 } \cline { 5 - 6 } & MoE & MoE-A & & MoE & MoE-A \\
\hline D,E,K-B & -0.70 & -0.51 & & -0.75 & -0.60 \\
B,E,K-D & -0.67 & -0.41 & & -0.05 & -1.20 \\
B,D,K-E & -1.93 & -0.44 & & -0.70 & -0.60 \\
B,D,E-K & -0.49 & -0.09 & & -0.50 & +0.30 \\
\hline
\end{tabular}

Table 3: Ablation test of entropy regularizer on CHEN 12 and ZISER 17 (decrease in accuracy).

on CHEN 12 and ZISER 17. It shows that entropy regularization benefits our model under both nonadversarial and adversarial settings.

\subsection{Part-of-Speech Tagging}

Table 4 summarizes our results on POS tagging. Again, our approach consistently achieves the best performance across different settings and tasks. Adding Twitter as a source leads to a drop in performance for the unified model, as a result of negative transfer. Our method, however, robustly handles negative transfer and manages to even benefit from this additional source.

Impact of negative transfer Table 5 presents the $\alpha$ distribution learned by the metric, on average for all tokens of the target domain. As we can see, our model (MoE-A) effectively learns to de- crease the weights on Twitter, demonstrating again its ability to alleviate negative transfer.

We further study the impact of this outlier source by varying the amount of Twitter data used during training. We gradually increase the number of Twitter instances by 750. As shown in Table 6, the increase of the Twitter data does not benefit the unified multi-source model (uni-MS-A), and even amplifies negative transfer for the Answers and Reviews domains. However, the performance of our MoE (MoE-A) model stays stable, consistently increasing with more Twitter, showing robustness in handling negative transfer.

\section{Conclusion}

In this paper, we propose a novel mixture-ofexperts (MoE) approach for unsupervised domain adaptation from multiple diverse source domains. We model the domain relations through a point-to-set distance metric, and introduce a meta-training mechanism to learn this metric. Experimental results on sentiment classification and part-of-speech tagging demonstrate that our approach consistently outperforms various baselines and can robustly handle negative transfer. The effectiveness of our approach suggests its potential application to a broader range of domain adaptation tasks in NLP and other areas. 


\begin{tabular}{|c|c|c|c|c|c|c|c|c|}
\hline \multirow{2}{*}{ TARGET } & \multicolumn{4}{|c|}{ NON-ADVERSARIAL } & \multicolumn{4}{|c|}{ ADVERSARIAL } \\
\hline & best-SS & uni-MS & uni-MS ${ }^{\dagger}$ & $\mathrm{MoE}$ & best-SS-A & uni-MS-A & uni-MS-A $^{\dagger}$ & MoE-A \\
\hline Answers & 88.16 & 88.89 & 89.88 & 90.26 & 88.47 & 89.04 & 89.99 & 89.80 \\
\hline Reviews & 87.15 & 87.45 & 88.91 & 89.37 & 87.26 & 87.90 & 88.94 & 89.40 \\
\hline Newsgroup & 89.14 & 89.95 & 90.70 & 91.03 & 89.54 & 90.20 & 90.70 & 91.13 \\
\hline Average & 88.15 & 88.76 & 89.83 & 90.22 & 88.42 & 89.05 & 89.88 & 90.11 \\
\hline
\end{tabular}

Table 4: POS tagging results on SANCL data. Source domains include Web, Emails, Twitter. ${ }^{\dagger}$ indicates the unified multi-source model trained without Twitter, thus can be considered as the oracle performance (upper-bound) of uni-MS.

\begin{tabular}{lccc}
\hline \multirow{2}{*}{ TARGET } & \multicolumn{3}{c}{ SOURCE } \\
\cline { 2 - 4 } & Twitter & Emails & Web \\
\hline Answers & 0.0527 & 0.5941 & 0.3531 \\
Reviews & 0.0640 & 0.5250 & 0.4100 \\
Newsgroup & 0.0538 & 0.4960 & 0.4490 \\
\hline
\end{tabular}

Table 5: Distribution of the metric values $\alpha$ on average for all tokens in the SANCL test set.

\begin{tabular}{llccc}
\hline \multirow{2}{*}{ TARGET } & ModEL & \multicolumn{3}{c}{ \# Twitter instances } \\
\cline { 3 - 5 } & $(*-\mathbf{A})$ & 750 & 1,500 & 2,250 \\
\hline \multirow{2}{*}{ Answers } & uni-MS & 89.04 & 89.04 & 86.93 \\
& MoE & 89.80 & 91.22 & 90.90 \\
\cline { 2 - 5 } Reviews & uni-MS & 87.90 & 87.45 & 87.68 \\
& MoE & 89.40 & 90.23 & 91.14 \\
\cline { 2 - 5 } Newsgroup & uni-MS & 90.20 & 90.10 & 90.21 \\
& MoE & 91.13 & 91.32 & 91.82 \\
\hline
\end{tabular}

Table 6: POS tagging accuracy with varying amounts of Twitter data in training.

\section{Acknowledgments}

We thank MIT NLP group and the anonymous reviewers for their helpful comments. We also thank Shiyu Chang and Mo Yu for insightful discussions on metric learning. This work is supported by the MIT-IBM Watson AI Lab. Any opinions, findings, conclusions, or recommendations expressed in this paper are those of the authors, and do not necessarily reflect the views of the funding organizations.

\section{References}

Shai Ben-David, John Blitzer, Koby Crammer, and Fernando Pereira. 2007. Analysis of representations for domain adaptation. In Advances in neural information processing systems. pages 137-144.

Himanshu Sharad Bhatt, Manjira Sinha, and Shourya Roy. 2016. Cross-domain text classification with multiple domains and disparate label sets. In Proceedings of the 54th ACL. pages 1641-1650.

John Blitzer, Mark Dredze, and Fernando Pereira. 2007. Biographies, bollywood, boom-boxes and blenders: Domain adaptation for sentiment classification. In Proceedings of the 45th ACL. Prague, Czech Republic, pages 440-447.

John Blitzer, Ryan McDonald, and Fernando Pereira. 2006. Domain adaptation with structural correspondence learning. In $E M N L P$. pages 120-128.

Konstantinos Bousmalis, George Trigeorgis, Nathan Silberman, Dilip Krishnan, and Dumitru Erhan. 2016. Domain separation networks. In Advances in Neural Information Processing Systems. pages 343351.

Rich Caruana. 1997. Multitask learning. Machine learning 28(1):41-75.

Minmin Chen, Zhixiang Xu, Kilian Q. Weinberger, and Fei Sha. 2012. Marginalized denoising autoencoders for domain adaptation. In Proceedings of the 29th International Conference on Machine Learning. Omnipress, USA, pages 1627-1634.

Xilun Chen and Claire Cardie. 2018. Multinomial adversarial networks for multi-domain text classification. arXiv preprint arXiv:1802.05694 .

Koby Crammer, Michael Kearns, and Jennifer Wortman. 2008. Learning from multiple sources. Journal of Machine Learning Research 9(Aug):17571774.

Lixin Duan, Ivor $\mathrm{W}$ Tsang, Dong $\mathrm{Xu}$, and Tat-Seng Chua. 2009. Domain adaptation from multiple sources via auxiliary classifiers. In Proceedings of the 26th International Conference on Machine Learning. ACM, pages 289-296.

Yaroslav Ganin, Evgeniya Ustinova, Hana Ajakan, Pascal Germain, Hugo Larochelle, François Laviolette, Mario Marchand, and Victor Lempitsky. 
2016. Domain-adversarial training of neural networks. The Journal of Machine Learning Research 17(1):2096-2030.

Xavier Glorot, Antoine Bordes, and Yoshua Bengio. 2011. Domain adaptation for large-scale sentiment classification: A deep learning approach. In Proceedings of the 28th international conference on machine learning. pages 513-520.

Arthur Gretton, Karsten M Borgwardt, Malte J Rasch, Bernhard Schölkopf, and Alexander Smola. 2012. A kernel two-sample test. Journal of Machine Learning Research 13(Mar):723-773.

Robert A Jacobs, Michael I Jordan, and Andrew G Barto. 1991a. Task decomposition through competition in a modular connectionist architecture: The what and where vision tasks. Cognitive science 15(2):219-250.

Robert A Jacobs, Michael I Jordan, Steven J Nowlan, and Geoffrey E Hinton. 1991b. Adaptive mixtures of local experts. Neural computation 3(1):79-87.

Yoon Kim. 2014. Convolutional neural networks for sentence classification. In Proceedings of EMNLP. Doha, Qatar, pages 1746-1751.

Young-Bum Kim, Karl Stratos, and Dongchan Kim. 2017. Domain attention with an ensemble of experts. In Proceedings of the 55th ACL. volume 1, pages 643-653.

Shoushan Li and Chengqing Zong. 2008. Multidomain sentiment classification. In Proceedings of the 46th ACL. pages 257-260.

Yijia Liu, Yi Zhu, Wanxiang Che, Bing Qin, Nathan Schneider, and Noah A Smith. 2018. Parsing tweets into universal dependencies. In NAACL.

Ping Luo, Fuzhen Zhuang, Hui Xiong, Yuhong Xiong, and Qing He. 2008. Transfer learning from multiple source domains via consensus regularization. In Proceedings of the 17th ACM conference on Information and knowledge management. ACM, pages 103-112.

Yishay Mansour, Mehryar Mohri, and Afshin Rostamizadeh. 2009. Domain adaptation with multiple sources. In Advances in neural information processing systems. pages 1041-1048.

Sinno Jialin Pan, Xiaochuan Ni, Jian-Tao Sun, Qiang Yang, and Zheng Chen. 2010. Cross-domain sentiment classification via spectral feature alignment. In Proceedings of the 19th international conference on World wide web. ACM, pages 751-760.

Slav Petrov, Dipanjan Das, and Ryan McDonald. 2012. A universal part-of-speech tagset. In Proceedings of the Eighth International Conference on Language Resources and Evaluation (LREC-2012). Istanbul, Turkey, pages 2089-2096.
Slav Petrov and Ryan McDonald. 2012. Overview of the 2012 shared task on parsing the web. In Notes of the first workshop on syntactic analysis of noncanonical language (sancl). volume 59.

Michael T Rosenstein, Zvika Marx, Leslie Pack Kaelbling, and Thomas G Dietterich. 2005. To transfer or not to transfer. In NIPS 2005 workshop on transfer learning. volume 898, pages 1-4.

Noam Shazeer, Azalia Mirhoseini, Krzysztof Maziarz, Andy Davis, Quoc Le, Geoffrey Hinton, and Jeff Dean. 2017. Outrageously large neural networks: The sparsely-gated mixture-of-experts layer. arXiv preprint arXiv:1701.06538.

Jian Shen, Yanru Qu, Weinan Zhang, and Yong Yu. 2018. Wasserstein distance guided representation learning for domain adaptation. In $A A A I$.

Jake Snell, Kevin Swersky, and Richard Zemel. 2017. Prototypical networks for few-shot learning. In $\mathrm{Ad}$ vances in Neural Information Processing Systems. pages 4080-4090.

Eric Tzeng, Judy Hoffman, Ning Zhang, Kate Saenko, and Trevor Darrell. 2014. Deep domain confusion: Maximizing for domain invariance. arXiv preprint arXiv:1412.3474 .

Oriol Vinyals, Charles Blundell, Tim Lillicrap, Daan Wierstra, et al. 2016. Matching networks for one shot learning. In Advances in Neural Information Processing Systems. pages 3630-3638.

Kilian Q Weinberger and Lawrence K Saul. 2009. Distance metric learning for large margin nearest neighbor classification. Journal of Machine Learning Research 10(Feb):207-244.

Jun Yang, Rong Yan, and Alexander G Hauptmann. 2007. Cross-domain video concept detection using adaptive svms. In Proceedings of the 15th ACM international conference on Multimedia. ACM, pages 188-197.

Mo Yu, Xiaoxiao Guo, Jinfeng Yi, Shiyu Chang, Potdar Saloni, Yu Cheng, Gerald Tesauro, Haoyu Wang, and Bowen Zhou. 2018. Diverse few-shot text classification with multiple metrics. In NAACL.

Yuan Zhang, Regina Barzilay, and Tommi Jaakkola. 2017. Aspect-augmented adversarial networks for domain adaptation. Transactions of the Association for Computational Linguistics 5:515-528.

Han Zhao, Shanghang Zhang, Guanhang Wu, Geoffrey J Gordon, et al. 2018. Multiple source domain adaptation with adversarial learning. ICLR Workshop .

Yftah Ziser and Roi Reichart. 2017. Neural structural correspondence learning for domain adaptation. In Proceedings of the 21st Conference on Computational Natural Language Learning (CoNLL 2017). Vancouver, Canada, pages 400-410. 